\title{
A Novel Musculoskeletal Imbalance Identification Mechanism for Lower Body Analyzing Gait Cycle by Motion Tracking
}

\author{
Hiranthi Tennakoon \\ School of Computing University of Colombo, Clombo7, Sri Lanka \\ E-mail: hiranthithennakoon@gmail.com
}

\author{
Charitha Paranamana, Maheshya Weerasinghe, Damitha Sandaruwan, Kalpani Mahindaratne \\ School of Computing University of Colombo, Colombo 7, Sri Lanka \\ E-mail: cparanamana93@gmail.com,amw@ucsc.cmb.ac.lk,dsr@ucsc.cmb.ac.lk, radha.km87@gmail.com
}

Received: 26 October 2017; Accepted: 17 November 2017; Published: 08 March 2018

\begin{abstract}
Muscles in a human body consists of a pair. Musculoskeletal imbalances caused by repetitive usage of one part of the muscle in this pair and incorrect posture a human body takes on regular basis lead to severe injuries in terms of neuro musculoskeletal problems, hamstring strains, lower back tightness, repetitive stress injuries, altered movement patterns, postural dysfunctions, trapped nerves and etc. and both neurological and physical performances are severely affected when time progresses. In clinical domain, muscle imbalances are determined by gait and posture analysis, Movement analysis, Joint range of motion analysis and muscle length analysis which require expertise knowledge and experience. X-Rays and CT scans in the medical domain also require domain experts to interpret the results of a checkup. Kinect is a motion capturing device which is able to track human skeleton, its joints and body movements within its sensory range. The purpose of this research is to provide a mechanism to identify muscle imbalances based on gait analysis tracked via Kinect motion capture device by differentiate the deviation of healthy person's gait patterns. Primarily, the outcome of this study will be a self-identification method of human skeletal imbalance.
\end{abstract}

Index Terms - Gait cycle, Imbalance, musculoskeletal system, Graph comparison.

\section{INTRODUCTION}

To function properly as individuals, it is important to endow a healthy and a strong body. In order to provide the body with shape, protection and internal structure, human skeleton is a necessary component and for human skeleton to function, support of skeleton muscles is crucial. Human body consists muscles which mostly work as pairs to assist these functionalities. A muscle imbalance can be defined as one muscle from the pair getting stronger, longer and stiffer than the other. For the proper utilization of muscles, muscle balance is a vital concern. Disorders in the musculoskeletal system is an important health problem to the general public considering the sickness absence, work disability and health care cost.Since the human skeleton cannot be accessed directly like skin, hair or nails and a daily care routine cannot be carried out on the surface level, we have to gain professional consultancy to identify the 'what could go wrongs' pertaining to the skeleton system and answer the identified issues in a proper manner for its wellbeing.

The relationship of the lower body skeletal system is at a stronger rate than the upper body skeletal system. Therefore, higher injury rates are associated with the lower body [4], [5] and applicability of this study to the upper body will be determined only if the approach can be applied to the lower body. Gait analysis is a new biometric technique which can be used to identify behavioral specifics of different people [6]. Usage of marker less systems is a technique which does not need physical contact between sensor and the subject to observe the gait cycle. Gait analysis is advantageous in allowing marker free identification, hard imitation and inability of hiding the postural patterns.

Considering marker-free systems, (i.e., no sensors or other devices are placed on the subject), gait recognition is an unintrusive technique, meaning that no physical contact between subject and measurement device is necessary. In marker-free gait recognition, person identification is usually executed by analyzing video sequence recordings [25]. According to recent cites Microsoft Kinect which is originally used as a video gaming device can be also used as a tool to track human gait variable as well as gait kinematics. Kinect is capable of tracking and recording 3D human motion without using markers [7], detects human motions in 3D view real time and extract artificial skeleton with joints overtime.

\section{A. Clinical analysis mechanisms}

- $\quad$ Gait cycle Analysis 
Gait cycle, primarily can be defined as the way a human walks; the cycle of phases from the initial contact of one foot with the ground and the next contact of the same foot with the ground. In gait analysis, physiotherapist observes the gait of an individual, analyses gathered information by comparing with standards and predicts the condition of the muscle. Gait cycle can be divided into two phases; stance and swing. A walkway of at least six meters or a treadmill is used to perform gait assessment. There is no one correct manner of assessing the gait and the best time to examine a patient's gait cycle is as they enter the examination room since they walk in naturally [3]. The following figure shows the events of a complete gait cycle.

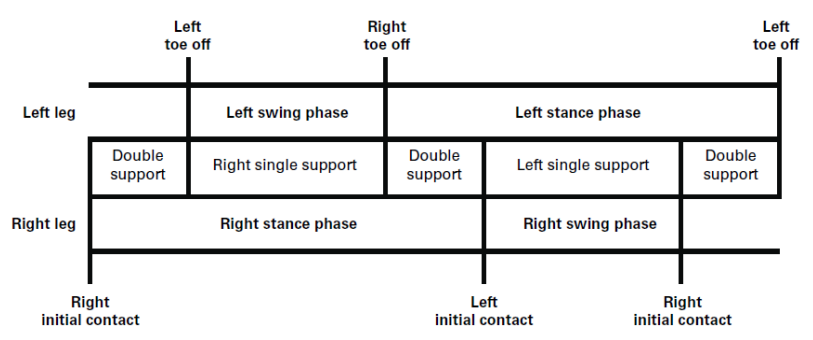

Fig.1. Gait cycle phases

\section{B. Movement Analysis}

In movement analysis, a patient is requested by the physiotherapist to perform various activities to determine whether the patient holds a musculoskeletal imbalance or not by analyzing the behaviors and stances the patient's body takes. Requested stance is highly depended on the experience and knowledge of the observer. There are three main methods of movement analysis; movement phases, free body diagrams and deterministic models. In movement analysis phase, analysis of preparation, execution and follow-through is analysed. In preparation phase, movement preparation by the individual to perform a particular motion is analysed and in the execution phase, movement of the patient is analysed. All the behaviors of the individual after the movement is also analysed in this phase.

\section{Joint Range of Motion Analysis}

In joint range of motion analysis, the range a human can perform his motions and movements is taken to consideration. Main considerations in this approach are; the plane of the movement occurrence, muscle used to produce the movement, function of the involved muscle; agonist [14]. There are mainly four planes a human body can be divided into;

In this analysis method all mentioned planes and axis and both active and passive range of motions are measured [14] and with the aid of Goniometer angles between the joints are measured and compared with the standard angles defined in the domain with respect to the person's age, weight, fat element. A noticeable deviation of joint angles from the standards is determined as a muscle imbalance.
4 Planes
- Sagittal plane
- Coronal plane
- Transverse plane
- Oblique plane
- Frontal axis
- Sagittal/ Transverse axis
- Longitudinal axis
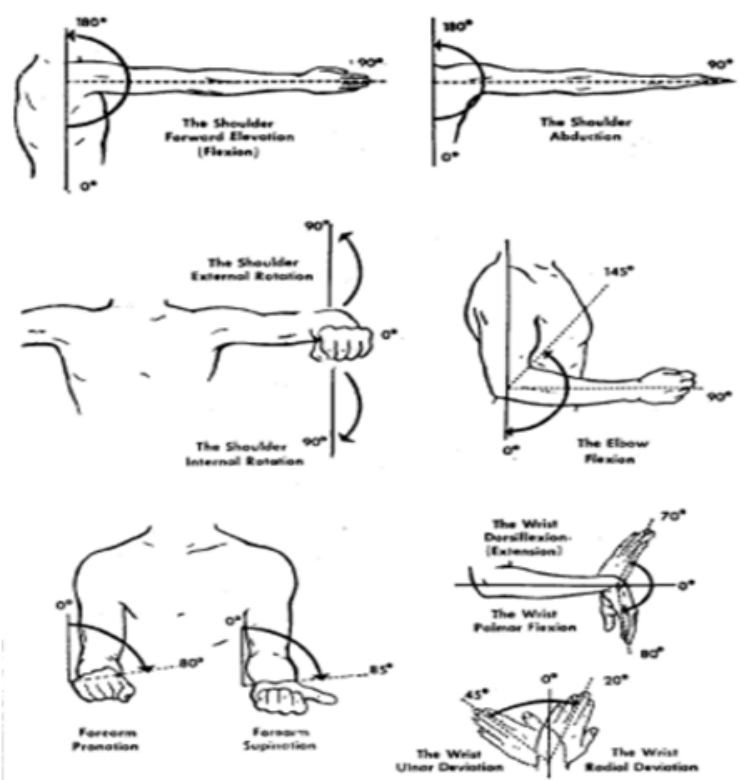

Fig.2. Main four planes

\section{Muscle Length Analysis}

Muscle length is analysed against the age and other physical parameters of the person which assesses the resistance to passive movement. In order to gain accurate result, muscle length should be performed when the patient is not in acute pain in order to avoid pain inhibition and muscle guarding [15]. Goniometer or a tape can be used to do the measurements and there are four steps in performing muscle length analysis [16].

- Ensure maximal lengthening of the muscle from origin to insertion

- Firmly stabilize one end (usually the origin)

- Slowly elongate the muscle

- Assess the end feel

\section{E. Technical Analysis Mechanisms}

\section{- Electromyography}

A needle is injected to the surface level of the human body and this stimulation is used to measure the reaction and activities of muscles to the mentioned electrical stimulation. Muscle activities grasped by the electrodes are displayed in a monitor called oscilloscope and an audio amplifier is also used to listen to the muscle activities. To determine a muscle imbalance, currently consultants analyse these muscle activities and their responses to the electric stimulation and a muscle imbalance is determined if found. 


\section{- X-Ray and CT Scan}

In Computed Tomography a special X-Ray equipment is used to detect body conditions. This scanning produces multiple images of the inner body of a person. X-Ray beams and electronic X-Ray detectors rotate around the patient and different parts of the body absorb the produced X-ray in different degrees and it records in a special electronic recording plate [17]. These record and datasets create a two dimensional image of the body. In determining musculoskeletal imbalances, the domain experts analyse these scanned body images to conclude whether the patient is having a muscle imbalance or not.

\section{RELATED WORKS}

After conducting an initial background study about the problem and the current imbalance identification mechanisms, we could find that there are issues in current physiotherapists' approach such as precision, cost, the domain knowledge required and time. Hence this literature survey was thoroughly conducted to identify a gap to build the solution. This survey is done under main three areas; the severity of muscle imbalance, the sensor or tracking devices which have been used to capture human motion tracking so far in researches and application of gait cycle in research domain.

\section{A. Muscle imbalance}

In recent years, clinical researches have been conducted to identify after effects of muscle imbalances and was found that the root cause for neuro musculoskeletal damages, lower back tightness, repetitive stress injuries, hamstring strains, altered movement patterns and postural dysfunctions lies in muscle imbalances [33], [34], [14]. It also depicted that a large range of motions of joints would lead to several causes like muscle over lengthening and imbalances in agonist and antagonist (a muscle pair) in [34]. The previous study also has concluded that frequent muscle over lengthening causes micro-trauma accumulation which can be resulted in repetitive stress injuries in long term. It is stated in a previous study [33] that faulty postures, joint misalignment and development of altered inefficient movement patterns result in strain and degeneration of joint, mofascial and ligamentous structures which lead to muscle imbalances. In [35] it is statistically given that there is a trend for higher injury rates to be associated with knee or hip extensor imbalances of $15 \%$ or more than on either side of the body. American journal of Sports Medicine in 2012 by conducting a research [35] on soccer players stated that there were 2123 muscle injuries documented in the major lower extremity muscle groups: adductors $(\mathrm{n}=523)$, hamstrings $(\mathrm{n}=900)$, quadriceps ( $\mathrm{n}$ $=394)$, and calf $(\mathrm{n}=306)$. Injuries to the adductors $(56 \%$; $\mathrm{P}=.015)$ and quadriceps $(63 \% ; \mathrm{P}<.001)$ were more frequent in the kicking leg of soccer players.

B. Utilization of motion tracking devices/ sensors for human movement capturing
In the IT domain researchers have done studies to identify muscle abnormalities using motion tracking sensors. In many studies they have considered sports domain to identify musculoskeletal issues since sports persons are often having issues in musculoskeletal system. Characteristics of the range of motions of the musculoskeletal system, muscles at risks and injury prevention strategies have been identified by using 3D motion tracking systems in a previous researches [3]. A nine-camera, 3D motion capturing system and 42 reflective markers on subject's each part of the body were used to track body movements and to identify muscles at risk. Using the raw 3D data captured from the ninecamera and 42 reflective markers, biomechanical modeling of the subject is produced and muscles and risks have been identified using this biomechanical model. Inertial tracking sensor which combines the signals from 3D gyroscopes, accelerometers and magnetometers and estimate the free orientation of human have also been used in previous studies [14].

When calculating human kinematics, it is required to define all joint orientation in its local frame so that local orientation can be easily converted into the angular values of each joint of musculoskeletal system [5]. In the inertial sensor, each individual sensor of the system provides orientation with respect to the global frame. The MVN motion capture system is consisted of body worn sensors which are having unique approach to estimate body segment orientation and position changes by integration of gyroscope and accelerometer signals which are continuously updated by using the biomechanical models of the human body which allows to track the human musculoskeletal system's motions [14].

Using biomechanical model which assumes that a subject's body includes body segments linked by joints and that the sensors are attached to the subject's body segments, the inertial navigation system kinematics are translated to body kinematics. When calculating the joint angles, the origin values are defined in the center of the functional axis with the directions of the $\mathrm{X}, \mathrm{Y}$ and $\mathrm{Z}$ related to the functional movements. MVN MotionGrid is an ultra-wideband based tracking technology complementary to the MVN system which is added a local GPS system to the sensor and capable of assessing all the kinematics of an athlete accurately even during high centripetal accelerations [14].

Kinect, a markerless technology has been used to study the precision in the joint angles computation against a professional optical motion capturing device. The researches have obtained a guaranteed range of disparities to make validations on the used two devices regarding joint angles when the limbs are involved [18]. Motion tracking devices as in Kinect since it is relatively a cost effective motion capturing device, introduces the possibility of changing paradigms based on complex and expensive technologies and devices in the biomechanical domain [19]. Human musculoskeletal motions can be tracked using Kinect in model-based and appearancebased approaches [19]. In model based approach, it provides a continuum of solutions which are costly and 
also depending on the wealth of visual information provided by a multi camera system. When it comes to appearance-based method, it is associated with much less computational cost and the complexity of hardware is very less. This model [19] minimizes the inconsistency between the 3D structure and appearance of hypothesized 3D model and the actual visual observation comes from the Kinect sensor.

When tracking the human motions using Kinect, it is possible to acquire images using the sensor with its accompanied depth map [20]. Skin color of the human body section can be detected followed by depth segmentation and also this is used to capture the 3D image of particular body segment. In a previous research[20] human hand has been tracked using the Kinect. In order to model each hand pose they have used 27 parameters represented as vectors. In order to quantify the inconsistency between hand hypotheses and the actual observation, it is possible to use graphics rendering techniques to compare skin and depth maps for a particular hand pose.

\section{Gait cycle applications}

In recent studies human gait cycle has been used to denote different type of outcomes of an individual. Analyzing the human gait cycle is a new emerging trend in the research world for many applications such as human identification, neurological disease identification and fall detection of elderly people.

Individual human recognition by gait cycle has been performed [30] via Gait Energy Image (GEI) mechanism. GEI is a spatio-temporal gait representation for individual recognition by characterizing the human gait cycle properties which is represented in a single image while preserving temporal information. This representation saves both storage and computation time for recognition compared to binary silhouette [26].

In recent studies gait cycle parameters have been used to be predictive of future falls and adverse events in older people using Kinect [28], [29], [30]. Impairments in gait and balance causes falling of adults basically. Identification of these gait abnormalities is essential to early initiation of fall prevention strategy. By analyzing the stride time, stride symmetry, speed, age parameters of sample objects while they walking, previous research have found that accelerometers give accurate results of gait analysis of fallers and non fallers [30]. They have placed accelerometers in head, trunk and the lower body to capture the gait cycle characteristics. Not only accelerometers but in other study [32] microsensors have been used to track the gait cycle and send the status of gait cycle to aid station real time at the falling moment. This system is very powerful to capture, analyze and communicate results to remote place real time. Kinect sensor with a computer deployed in an apartment was used in another study to capture the gait cycle of older adults in the apartment and make predictions on their health situation [29].
Identification of neurological disorders of patients was conducted based on the support, Double support time percentage, Single support time percentage, Step-length, Step-time, step-width, Stride-length, Stride-time, and Swing-time characteristics of gait cycle [27]. This research group was capable of identifying the gait variability of each neurologic patients for future prediction. By analyzing these characteristics, the certain disease was identified.

\section{RESEARCH DESIGN}

After the literature survey about the technical aspect and the research approach to the problem, what we understood was, analyzing the gait cycle is very emerged area in recent researches and using sensors and motion tracking devices to identify gait cycle is a trend in researches. But not one have conducted a research to identify musculeskeletal imbalance by analyzing the gait cycle. Hence our solution is analyzing human gait cycle to identify musculeskeletal imbalance of lower body using motion tracking device Kinect. Chapter III describes the design of the solution.

\section{A. Data gathering and sampling methodology}

\section{- Sample selection}

The sample of this research is consisted of both men and women. The male subjects fall between the age range of $25-45$. The female subjects falls between the age range of 30 to 50. This age limits are chosen as these ranges falls between skeletal cycle completion and depreciation periods [21]. The subjects of the sample were having normal BMI value of $18.5-23$ which indicates the BMI of a healthy person [22] and normal step size $5 \mathrm{~cm}-10 \mathrm{~cm}$ which is the step size of a person who falls under the age ranges specified [22], [23]. Individuals having diseases which affect the gait cycle have not been included in the sample of this research. The sample was selected with supervision of domain experts and subjects who suffers from musculoskeletal imbalances were used in this research to prove the accuracy and reliability of the proposed methodology of analyzing muscle imbalances. We provided written consents to the sample prior to their participation in the experiment as it is the ethical way of doing human based experiments.

\section{- Data gathering}

Prior to capturing the individual gait cycles, user details of the subject were fed through the initial interface of the application. User name, age, height, weight, gender and number of gait cycles which is going to be tracked are the data fed into the system. In order to increase the accuracy of the process, more than one gait cycle was always captured. Followed by the initial user detail fed, capturing the human skeleton and its movements were proceeded. 


\section{B. Mathematical model to capture joint angles from the Kinect}

In order to calculate the angles between the joints (hip, knee and ankle) using the captured joint points from the Kinect, following mathematical model was used.

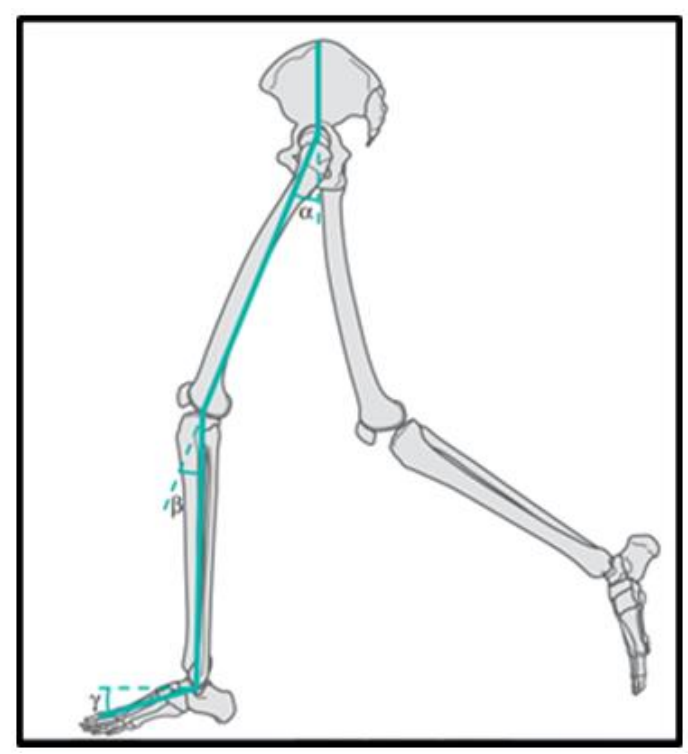

Fig.3. Three joint angles in lower limb

Hip, knee and ankle joint angles are denoted by $\mathrm{P}, \mathrm{Q}$ and $\mathrm{R}$. Dot product function which is the standard way to calculate the angle between two vectors in $3 \mathrm{D}$ space in mathematics is used to calculate the joint angles in 3D space.

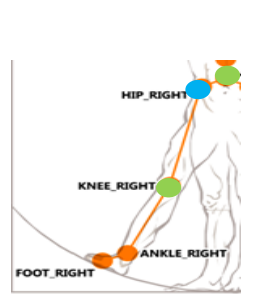

Fig.4.

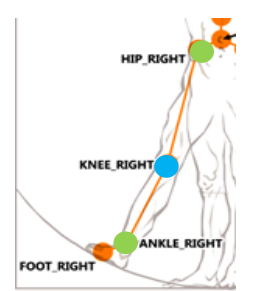

Fig.5.

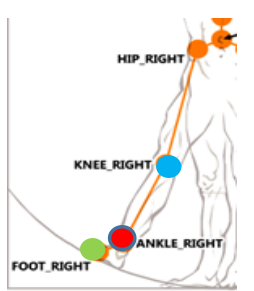

Fig.6.
As pointed out in Figure 1, blue circle shows the hip angle which we need to calculate and two green circles shows the other two related joints it considers when calculating the hip angle, hip center and the knee joint. Dot product function which calculates the angle between two vectors in $3 \mathrm{D}$ space is used in this research to calculate the relevant angles in the research.

\section{$\operatorname{Dot} \operatorname{product}(\mathrm{PQ}, \mathrm{QR})=\|\mathrm{PQ}\|\|\mathrm{QR}\| \cos \phi$}

\section{Step 1}

Join the three related joint consider and create two vectors in $3 \mathrm{D}$ space

Example: If we consider three joints are $\mathrm{P}, \mathrm{Q}, \mathrm{R}$ then two vectors are PQ and $\mathrm{QR}$

Let us assume the $\mathrm{x}, \mathrm{y}, \mathrm{Z}$ coordinates as follows

$$
\begin{gathered}
\mathrm{P}=(\mathrm{x} 1, \mathrm{y} 1, \mathrm{z} 1), \mathrm{Q}=(\mathrm{x} 2, \mathrm{y} 2, \mathrm{z} 2), \mathrm{R}=(\mathrm{x} 3, \mathrm{y} 3, \mathrm{z} 3) \\
\mathrm{PQ}=\mathrm{Q}-\mathrm{P} \rightarrow 1 \\
\mathrm{QR}=\mathrm{R}-\mathrm{Q} \rightarrow 2
\end{gathered}
$$

1. $P Q=((x 2-x 1),(y 2-y 1),(z 2-z 1)) \rightarrow$ Lets say $(X 1, Y 1, Z 1)$

2.

$\mathrm{QR}=((\mathrm{x} 3-\mathrm{x} 2),(\mathrm{y} 3-\mathrm{y} 2),(\mathrm{z} 3-\mathrm{z} 2)) \rightarrow$ Lets say $(\mathrm{X} 2, \mathrm{Y} 2, \mathrm{Z} 2)$ $1 * 2 \rightarrow \mathrm{PQ}$. QR $=(X 1)(X 1)+(Y 1)(Y 2)+(Z 1)(Z 2)$

Step 2

Since, $\mathrm{PQ}=(\mathrm{X} 1, \mathrm{Y} 1, \mathrm{Z} 1)$ and $\mathrm{QR}=(\mathrm{X} 2, \mathrm{Y} 2, \mathrm{Z} 2)$

$$
\begin{aligned}
& \|\mathrm{PQ}\|=\sqrt{ }(\mathrm{X} 1)^{2}+(\mathrm{Y} 1)^{2}+(\mathrm{Z} 1)^{2} \\
& \|\mathrm{QR}\|=\sqrt{ }(\mathrm{X} 2)^{2}+(\mathrm{Y} 2)^{2}+(\mathrm{Z} 2)^{2}
\end{aligned}
$$

Thus,

$$
\phi=\arccos \frac{(X 1)(X 1)+(Y 1)(Y 2)+(Z 1)(Z 2)}{(\sqrt{ }(X 1) 2+(Y 1) 2+(Z 1) 2)\left(\sqrt{ }(X 2)^{2}+(Y 2)^{2}+(Z 2)^{2}\right)}
$$

\section{CSV file generation}

When a subject walks in a straight line, the Kinect sensor would capture all the body joints and the application is developed to record and calculate the lower limb body joint angle values in each phase of the gait cycle. Hip, knee and the ankle angle values of both right and left side in every $200 \mathrm{~ms}$ is written to a .csv file and stored in the system. Number of .csv files which are generated is equal to the number of gait cycle values given in the initial data fed.

\section{Gait cycle graph}

Gait cycle graph represents how the joint angles change throughout one gait cycle. This graph can be drawn mainly in three different ways when it comes to lower limb musculoskeletal system; graph for hip variation in gait, graph for knee variation in gait, graph for ankle variation in gait. Gait cycle graph represents the angle of a particular joint in each phase of the gait cycle. $\mathrm{X}$ axis denotes the percentage of the gait cycle as $10 \%$, $20 \% \ldots 100 \%$ and $\mathrm{Y}$ axis denotes the angle of the particular joint. The time taken to complete a gait cycle by a healthy person is around two seconds. Hence it captures the joint angle values in each 200 milliseconds by the application which denotes $10 \%$ of the gait cycle. In order to generate this graph, joint angles in each phase of the gait cycle were captured with the aid of the application developed. The graph is then generated using the angle values in each stage of the gait cycle.

\section{- Knee gait cycle graph}

Knee gait cycle graph denotes how the knee joint angle is changed throughout each phase of the gait cycle. How the knee joint angle changes throughout the time of the 
gait cycle is drawn in knee gait cycle graph. In this graph, $\mathrm{x}$ axis denotes time and the $\mathrm{y}$ axis denotes the angle of knee joint.

\section{- Hip gait cycle graph}

Hip gait cycle graph denotes how the hip joint angle is changed throughout each phase of the gait cycle. How the hip joint angle changes throughout the time of the gait cycle is drawn in hip gait cycle graph. In this graph, $\mathrm{x}$ axis denotes time and the y axis denotes the angle of hip joint.

\section{- Ankle gait cycle graph}

Ankle gait cycle graph denotes how the ankle joint angle is changed throughout each phase of the gait cycle. How the ankle joint angle changes throughout the time of the gait cycle is drawn in ankle gait cycle graph. In this graph, $\mathrm{x}$ axis denotes time and the $\mathrm{y}$ axis denotes the angle of ankle joint.

\section{E. Generation of the standard gait cycle graph}

In order to draw the standard gait cycle graphs for hip, knee and ankle, a healthy sample is taken for the experiment. The sample is consisted of 10 male subjects and 10 female subjects. Hip, knee and the ankle joint angle values are taken from the .csv file generated and separate three graphs are created. Data gathering is done three times for each individual to increase the data accuracy and the average value of these three is taken. All male gait cycle graphs of hip is drawn and the minimum and maximum threshold values are determined by analyzing those data. Female minimum and maximum threshold values are also calculated in the same way. Knee and the ankle standard gait cycles also generated by following the same procedure.

\section{F. Graph comparison to identify Imbalance}

When an undefined subject comes for the experiment, after gathering the initial details, particular number of gait cycles are tracked by the system. Number of gait cycles that is captured can be vary according to the necessary level of accuracy. After the angle values are written to .CSV file, the average hip, knee and ankle gait cycle values of the subject is calculated by the application itself. Creating hip, knee and ankle gait cycle graphs for both right and left side is the next step in the process. The created three graphs are then compared with the standard gait cycle graphs separately in order to identify whether the subject is having an imbalance in his lower limb and where the imbalance is.

\section{CONCLUSION}

Previous identification of muscle imbalances is crucial for every individual to treat and overcome the situation in its early stages and avoid the occurrence of severe injuries. With the current mechanisms of identification lie many drawbacks. Muscle imbalances are not visible to the eye unless they rise with noticeable symptoms. Starting treatments for the imbalance in its latter stages will cause individuals to handle more developed neurological and physical dysfunctions.

A self-identification method is required in order to provide individuals with the ability to track their musculoskeletal system and treat it with proper care. Assessing muscle imbalances with the aid of technologies as in Kinect where human skeleton, gait cycle can be detected and by combining the medical domain with information technology and provide the physiotherapists and consultants with an aiding device, providing a selfidentification mechanism for human body imbalances is the primary objective of this research.

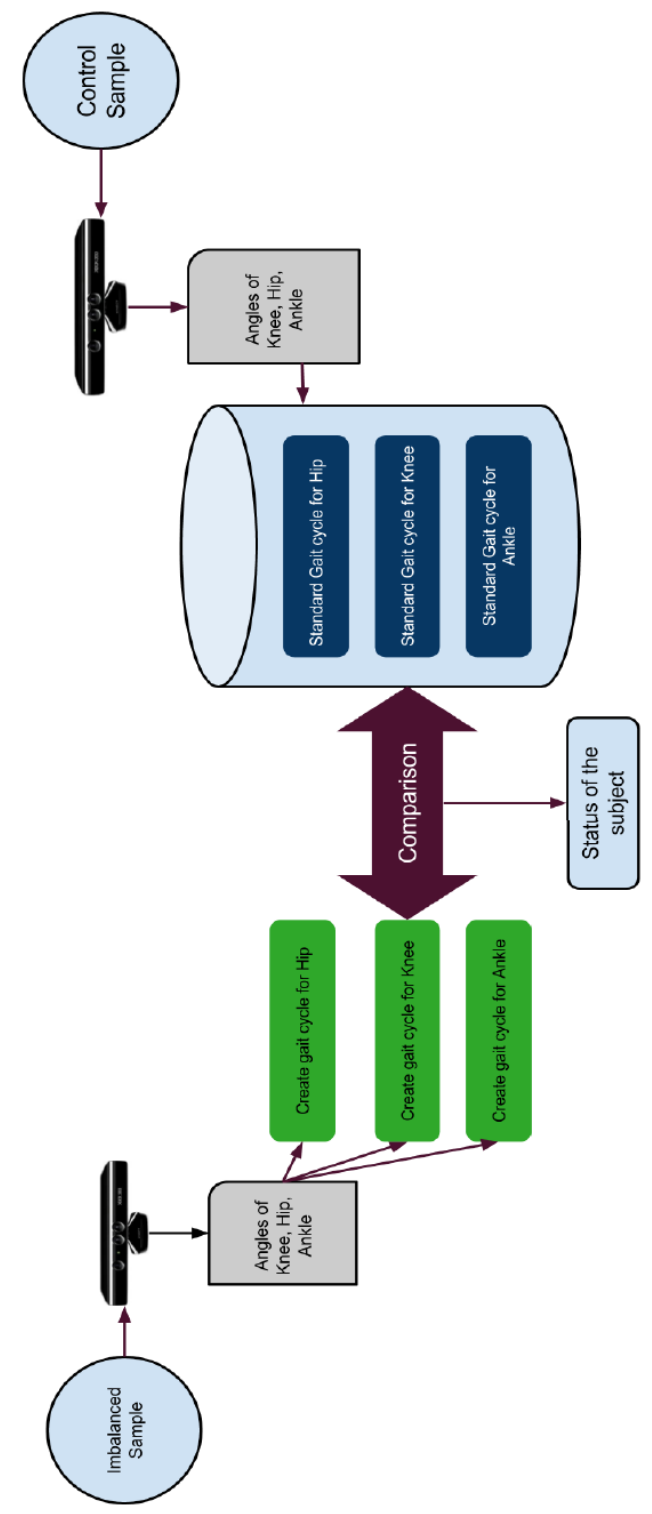

Fig.7. High level design

\section{REFERENCES}

[1] A. Pourmoghaddam, M. Dettmer, D. O'Connor, W. Paloski and C. Layne, "Identification of Changing Lower Limb Neuromuscular Activation in Parkinson's Disease during Treadmill Gait with and without Levodopa Using a Nonlinear Analysis Index", Parkinson's Disease, vol. 2015, pp. 1-8, 2015. 
[2] S. Perumal and R. Sankar, "Gait and tremor assessment for patients with Parkinson's disease using wearable sensors", ICT Express, vol. 2, no. 4, pp. 168-174, 2016

[3] Wan, Bingjun and Gongbing Shan. "Biomechanical Modeling As A Practical Tool For Predicting Injury Risk Related To Repetitive Muscle Lengthening During Learning And Training Of Human Complex Motor Skills". (2016): n. pag. Print.

[4] M. Hägglund, M. Waldén and J. Ekstrand, "Risk factors for lower extremity muscle injury in professional soccer: the UEFA Injury Study.", 2012. 23 Mar. 2017.]

[5] N. MILLER, O. JENKINS and M. KALLMANN, "MOTION CAPTURE FROM INERTIAL SENSING FOR UNTETHERED HUMANOID TELEOPERATION", 2004. 04.05.2017

[6] Preis, Johannes, Moritz Kessel, and Martin Werner. "Gait Recognition With Kinect." researchgate.net (2017): 1-4. Web. 18 July 2017.

[7] Springer, Shmuel, and Galit Yogev Seligmann. "Validity Of The Kinect For Gait Assessment: A Focused Review." semanticscholar (2016): n. pag. Web. 18 July 2017.

[8] 'Hugglund, Martin and Markes Walden. "Risk Factors For Lower Extremity Muscle Injury In Professional Soccer". Risk Factors for Lower Extremity Muscle Injury in Professional Soccer (2017): n. pag. Web. 13 Mar. 2017.

[9] T. ANDRIACCHI, M. HENRIKSEN and T. GRAVENNIELSEN, "Gait Changes in Patients With Knee OsteoarthritisAre Replicated by Experimental Knee Pain", Research Gate, vol. 62, no. 4, 2010.

[10] R. Bajcsy, "Analysis of the Effect of Cognitive Load on Gait with off-the-shelf Accelerometers", Research gate, 2017.

[11] C. Groner, "Diabetes and altered gait: The role of neuropathy | Lower Extremity Review Magazine", Lermagazine.com, 2017.

[12] J. Brach, J. Talkowski, E. Strotmeyer and A. Newman, "Diabetes Mellitus and Gait Dysfunction: Possible Explanatory Factors", Physical Therapy, vol. 88, no. 11, pp. 1365-1374, 2008.

[13] L. Allet, S. Armand, R. de Bie, A. Golay, D. Monnin, K. Aminian, J. Staal and E. de Bruin, "The gait and balance of patients with diabetes can be improved: a randomised controlled trial", Diabetologia, vol. 53, no. 3, pp. 458-466, 2009.

[14] D. Roetenberg, H. Luinge and P. Slycke, "Xsens MVN: Full 6DOF Human Motion Tracking Using Miniature Inertial Sensors", XSENS TECHNOLOGIES, 2003.

[15] A. Atrsaei, H. Salarieh and A. Alasty, "Human Arm Motion Tracking by Orientation-Based Fusion of Inertial Sensors and Kinect Using Unscented Kalman Filter", 2017.

[16] I. Oikonomidis and N. Kyriazis, "Efficient Model-based 3D Tracking of Hand Articulations using Kinect", 04.05.2017.

[17] Frank, Lardner and Page, "The assessment and treatment of muscular imbalance," The Janda Approach Hardback, Human Kinetics, Champlain, IL USA.

[18] Kenyon, Karen and Kenyon, Jonathan. The Physiotherapist's Pocket Book - Essential Facts At Your Fingertips. 2nd ed. Kundli (Haryana): Rajkamal Electric Press, 2017. Print.

[19] A. Fernandez, A. Susin and X. Lligadas, Biomechanical Validation of Upper-body and Lower-body Joint Movements of Kinect Motion Capture Data for Rehabilitation Treatments, 2012.
[20] I. Oikonomidis and N. Kyriazis, "Efficient Model-based 3D Tracking of Hand Articulations using Kinect", 04.05.2017

[21] Preis, J., Kessel, M., Werner, M. and Linnhoff-Popien, C. (2012). Gait recognition with Kinect. ResearchGate

[22] K. Culhane, "Accelerometers in rehabilitation medicine for older adults", Age and Ageing, vol. 34, no. 6, pp. 556560, 2005.

[23] Orthopedic for rehabilitation

[24] E. Carmeli, S. Kessel, R. Coleman and M. Ayalon, "Effects of a Treadmill Walking Program on Muscle Strength and Balance in Elderly People With Down Syndrome", 2002. Web. 23 Mar. 2017.

[25] Han, J. and Bhanu, B. (2006). Individual Recognition Using Gait Energy Image. IEEE

[26] M. Y, S. J, A. R, H. ME and S. JJI, "Gait variability in people with neurological disorders: A systematic review and meta-analysis", 2016.

[27] "Gait Analysis in Neurological Disease", Smart patients, 2016

[28] Stone, E. and Skubic, M. (2013). Unobtrusive, Continuous, In-Home Gait Measurement Using the Microsoft Kinect. IEEE, 60(10)

[29] K. Culhane, "Accelerometers in rehabilitation medicine for older adults", Age and Ageing, vol. 34, no. 6, pp. 556560, 2005.

[30] M. Hägglund, M. Waldén and J. Ekstrand, "Risk factors for lower extremity muscle injury in professional soccer: the UEFA Injury Study.", 2012.]

[31] C. Lin, H. Hsu, Y. Lay, C. Chiu and C. Chao, "Wearable device for real-time monitoring of human falls", Measurement, vol. 40, no. 9-10, pp. 831-840, 2007.

[32] S. K. and M. M., Gait and posture - assessment in general practice. 2017.

[33] Wan, Bingjun and Gongbing Shan. "Biomechanical Modeling As A Practical Tool For Predicting Injury Risk Related To Repetitive Muscle Lengthening During Learning And Training Of Human Complex Motor Skills". (2016): n. pag. Print

[34] Knapik and C. Bauman, "Preseason strength and flexibility imbalances associated with athletic injuries in female collegiate athletes.", 1991.

\section{Authors' Profiles}

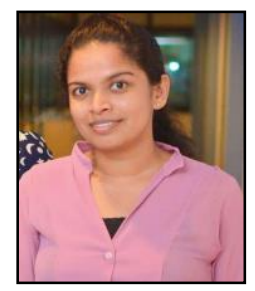

Hiranthi Tennakoon is a final year undergraduate of School of computing, University of Colombo Sri Lanka. She will be graduating in January 2018 in Information Systems Honors degree.

Her research interests are Modeling and simulation and Human computer Interaction

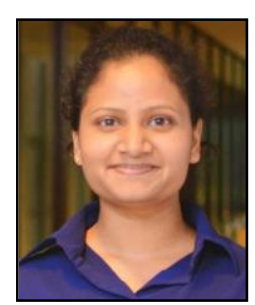

Charitha Paranamana is a final year undergraduate of School of Computing, University of Colombo Sri Lanka. She will be graduating in January 2018 in Information Systems Honors degree.

Her research interests are Computer Forensics, Simulation techniques and Human computer Interaction 


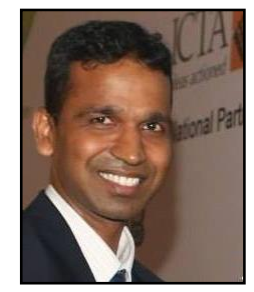

Damitha Sandaruwan received his BSc in Physical Science from the University of Colombo, Sri Lanka, PhD in Computer Science from University of Colombo School of Computing (2015).

Media Coordinator of the Advanced Digital Media Technology Centre (ADMTC) UCSC (2008-2014). Coinvestigator of the Modelling and Simulation Research Group, UCSC, Sri Lanka.

$\mathrm{He}$ is currently a Senior Lecturer Gr. II, at the UCSC, Sri Lanka. His Research Interests are Real-time Simulation, Modelling and Simulation, Computer Graphic and Vision.

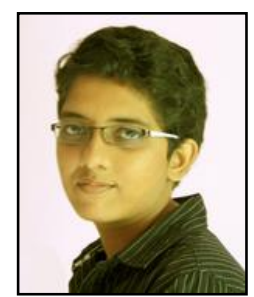

Maheshya Weerasinghe received her BSc in Computer Science (2014) from the University of Colombo School of Computing, Sri Lanka.

Student Member of the Computer Society and IEEE Student Branch of University of Colombo School of Computing (2010-2014). Member of the Modelling and Simulation Research Group, UCSC, Sri Lanka 2014-Present.

She is currently a Research Assistant, at the Modelling and Simulation Research Group, UCSC, Sri Lanka. Her Research Interests are Modelling and Simulation, Cognitive Neuroscience, Game based Learning, Cognitive Rehabilitation, Quantum Computing.

How to cite this paper: Hiranthi Tennakoon, Charitha Paranamana, Maheshya Weerasinghe, Damitha Sandaruwan, Kalpani Mahindaratne, "A Novel Musculoskeletal Imbalance Identification Mechanism for Lower Body Analyzing Gait Cycle by Motion Tracking", International Journal of Information Technology and Computer Science(IJITCS), Vol.10, No.3, pp.27-34, 2018. DOI: 10.5815/ijitcs.2018.03.04 